\title{
Controller Reduction of Parameter Dependent Systems
}

\author{
Widowati $^{*}$, Bambang Riyanto ${ }^{\dagger} \&$ Roberd Saragih ${ }^{\ddagger}$ \\ 1, ‡ Mathematics Department, Institut Teknologi Bandung, \\ Jl. Ganesha 10, Bandung 40132, Indonesia \\ ${ }^{\dagger}$ Electrical Engineering Department, Institut Teknologi Bandung, \\ Jl. Ganesha 10, Bandung 40132, Indonesia
}

\begin{abstract}
This paper proposes a controller reduction of linear parameter dependent systems. A measure of the degradation is derived for the parameter dependent closed-loop performance caused by applying the reduced-order parameter dependent controller. This measure can be obtained by extending the degradation of the closed-loop performance of the balanced truncation of the corresponding time invariant systems. To verify the performance of the reducedorder controller, an application of the proposed controller reduction method to vertical acceleration of a missile is presented.
\end{abstract}

Keywords: balanced truncation; closed-loop performance; parameter dependent controller; reduced-order controller.

\section{$1 \quad$ Introduction}

Almost all physical systems have parameter dependent representations, but many the current modelling and control of physical systems use Linear Time Invariant (LTI) systems. As operating conditions change, the behavior of the physical systems and the linear time invariant model vary so that the closed loop performance designed by LTI controller may degrade. To cope with this problem, it is required that the parameter dependent is designed using parameter dependent controller. Moreover, modern controller design theories such as $\mathcal{H}_{2}$ and $\mathcal{H}_{\infty}$ synthesis usually produce controllers that have the same order as that of the model. Thus, the application of these design techniques to high order models will produce high order controllers. The design and analysis of highorder controllers demand high computational cost and may results in numerical difficulties while its implementation is very complex. Hence, it is important to find the low (reduced)-order controllers for parameter dependent systems.

A reduced-order controller can be obtained through the direct method and the indirect method [1]. In the direct method, the reduced-order controller is

\footnotetext{
* Permanent address: Department of Mathematics, FMIPA, Diponegoro University, Kamp. Tembalang, Semarang, Indonesia.
} 
obtained directly from the high-order plant. In the indirect method, the reducedorder controller is obtained by using a controller reduction or a model reduction, i.e. a high-order controller is first found and then the order of the controller is reduced or a reduced-order plant is first found and then a reduced-order controller is designed.

Balanced Truncation (BT) method have been proposed to reduce both the order of the model and of the controller for LTI systems [7, 13]. The LTI model approximation techniques of BT method have been extended to reduce a Linear Time Varying (LTV) systems [10, 11]. Wood et.al [12] and Zobaidi et.al [6] generalized this method to reduce parameter dependent systems with unbounded rate parameter model and controller. They $[10,11,12]$ extend the twice the sum of the tail formula well known in the LTI case. In this paper we will find a reduced-order parameter dependent controller via a controller reduction. In comparison with Zobaidi et.al [6] method, it is different in the controller design technique. Zobaidi [6] et.al use solutions of the control and filtering riccati inequalities to construct a full-order parameter dependent controller, whereas in this paper it is constructed from the solutions of the linear matrix inequalities developed by Apkarian [2]. We also investigate the degradation of the parameter dependent closed-loop performance in $\mathcal{L}_{2}$ norm due to the reduced-controller. Furthermore, simulation results are given to examine the performance of the reduced-order parameter dependent controller for a missile.

\section{Preliminaries and Problem Formulation}

In this paper, we consider linear parameter dependent systems. For a compact subset $\mathcal{P} \subset \mathcal{R}^{s}$, the parameter variation set $\mathcal{F}_{\rho}$ denotes the set of all piecewise continuous mapping $\mathcal{R}$ (time) into $\mathcal{P}$ with a finite number of discontinuity in any interval. $\mathcal{F}_{\rho}:=\left\{\rho(t): \mathcal{R} \rightarrow \mathcal{R}^{s}, \rho_{i_{\min }} \leq \rho_{i} \leq \rho_{i_{\max }}, i=1,2, \ldots, s\right\}$. A compact set $\mathcal{P} \subset \mathcal{R}^{s}$, along with continuous functions $A: \mathcal{R}^{s} \rightarrow \mathcal{R}^{n \times n}, \quad B: \mathcal{R}^{s} \rightarrow \mathcal{R}^{n \times n_{u}}$, $C: \mathcal{R}^{s} \rightarrow \mathcal{R}^{n_{y} \times n}, \quad B: \mathcal{R}^{s} \rightarrow \mathcal{R}^{n_{y} \times n_{u}}$ represent an $n$ th-order parameter dependent plant, $G(\rho)$, whose dynamics evolve as

$$
\begin{aligned}
& \dot{x}(t)=A(\rho(t)) x(t)+B(\rho(t)) u(t) \\
& y(t)=C(\rho(t)) x(t)+D(\rho(t)) u(t), \forall \rho(t) \in \mathcal{F}_{\rho} .
\end{aligned}
$$

A state space realization of the parameter dependent plant, $G(\rho)$, is written as 


$$
G(\rho)=\left[\begin{array}{l|l}
A(\rho(t)) & B(\rho(t)) \\
\hline C(\rho(t)) & D(\rho(t))
\end{array}\right], \forall \rho(t) \in \mathcal{F}_{\rho} .
$$

The parameter dependent systems $G_{\rho}$ is quadratically stable $[9,12]$ if there exits a real positive-definite matrix $P=P^{T}>0$ such that

$$
A^{T}(\rho(t)) P+P A(\rho(t))<0, \forall \rho(t) \in \mathcal{F}_{\rho} .
$$

The induced $\mathcal{L}_{2}$ norm of a quadratically stable parameter dependent systems, $G(\rho)$, with zero initial conditions, is defined as [3]

$$
\|G(\rho)\|_{i, 2}=\sup _{\rho(t) \in \mathcal{F}_{\rho}} \sup _{u \neq 0, u \in \mathcal{L}_{2}} \frac{\|y\|_{2}}{\|u\|_{2}} .
$$

This quantity is always finite. To formulate a performance oriented parameter dependent output feedback synthesis problem, the parameter dependent plant is written as follows

$$
\begin{aligned}
& \dot{x}(t)=A(\rho(t)) x(t)+B_{1}(\rho(t)) w(t)+B_{2}(\rho(t)) u(t), \\
& z(t)=C_{1}(\rho(t)) x(t)+D_{11}(\rho(t)) w(t)+D_{12}(\rho(t)) u(t), \\
& y(t)=C_{2}(\rho(t)) x(t)+D_{21}(\rho(t)) w(t)+D_{22}(\rho(t)) u(t), \quad \forall \rho(t) \in \mathcal{F}_{\rho} .
\end{aligned}
$$

Readers are referred to references $[9,12]$ for the definition of the quadratic stabilizability and quadratic detectability. The following assumptions are made to parameter dependent plant

1. $D_{22}(\rho(t))=0$

2. $B_{2}(\rho(t)), C_{2}(\rho(t)), D_{12}(\rho(t))$, and $D_{21}(\rho(t))$ are parameterindependent

3. The pairs $\left(A(\rho(t)), C_{2}\right)$ and $\left(A(\rho(t)), C_{2}\right)$ are quadratically stabilizable and quadratically detectable over $\mathcal{P}$ respectively.

A construction of full-order controller has been developed by Apkarian, et.al [2]. The design objectives are to satisfy $\mathcal{H}_{\infty}$ performance criterion, i.e., the parameter dependent closed-loop systems is quadratically stable over $\mathcal{P}$ and the $\mathcal{L}_{2}$ gain of the parameter dependent closed-loop systems is bounded by $\gamma, \gamma>0$ for all possible trajectories $\rho$. In this paper we assume that the fullorder controller has an $m$ th-order. The parameter dependent full-order controller has state space realization as follows. For brevity, $t$ is omitted. 


$$
K(\rho)=\left[\begin{array}{c|c}
A_{K}(\rho) & B_{K}(\rho) \\
\hline C_{K}(\rho) & D_{K}(\rho)
\end{array}\right], \forall \rho \in \mathcal{F}_{\rho} .
$$

The goal of this paper is to find a reduced-order controller $\hat{K}(\rho)$ by using balanced truncation with state space realization given by

$$
\hat{K}(\rho)=\left[\begin{array}{c|c}
A_{k 1}(\rho) & B_{k 1}(\rho) \\
\hline C_{k 1}(\rho) & D_{k 1}(\rho)
\end{array}\right], \forall \rho \in \mathcal{F}_{\rho} .
$$

such that the degradation of the parameter dependent closed-loop performance $\left\|T_{z w}(\rho)-\hat{T}_{z w}(\rho)\right\|_{i, 2}$ is small, or at least bounded by an apriori bound, where $T_{z w}(\rho)$ and $\hat{T}_{z w}(\rho)$ are the parameter dependent closed-loop systems with the full-and reduced-order parameter dependent controllers, respectively.

\section{Order Reduction of the Parameter Dependent Controller}

Our aim in this section, is to extend some of the standard results on balanced truncation method of time invariant systems to controller reduction of the unbounded-rate parameter dependent systems. The realization of the parameter dependent closed-loop system with the full-order controller, $K(\rho)$ in equation 4, can be expressed by

$$
T_{z w}(\rho)=\left[\begin{array}{c|c}
\bar{A}(\rho) & \bar{B}(\rho) \\
\hline \bar{C}(\rho) & \bar{D}(\rho)
\end{array}\right], \forall \rho \in \mathcal{F}_{\rho},
$$

where

$$
\begin{aligned}
& \bar{A}(\rho)=\left[\begin{array}{cc}
A(\rho)+B_{2} D_{K}(\rho) C_{2} & B_{2} C_{K}(\rho) \\
B_{K}(\rho) C_{2} & A_{K}(\rho)
\end{array}\right], \bar{B}(\rho)=\left[\begin{array}{c}
B_{1}(\rho)+B_{2} D_{K}(\rho) D_{21} \\
B_{K}(\rho) D_{21}
\end{array}\right], \\
& \bar{C}(\rho)=\left[\begin{array}{ll}
C_{1}(\rho)+D_{12} D_{K}(\rho) C_{2} & \left.D_{12} C_{K}(\rho)\right], \bar{D}=D_{11}(\rho)+D_{12} D_{K}(\rho) D_{21} .
\end{array}\right.
\end{aligned}
$$

Assume that the full-order controller, $K(\rho)$ in equation 4, is a stabilizing parameter dependent controller so that $T_{z w}(\rho)$ is quadratically stable over $\mathcal{P}$ and that there exist $U=\operatorname{diag}\left(U_{1}, U_{2}\right)>0$ and $V=\operatorname{diag}\left(V_{1}, V_{2}\right)>0$ such that $\forall \rho \in \mathcal{F}_{\rho}$,

$$
\begin{aligned}
& \bar{A}(\rho) U+U \bar{A}^{T}(\rho)+\bar{B}(\rho) \bar{B}^{T}(\rho)<0 \\
& \bar{A}^{T}(\rho) V+V \bar{A}(\rho)+\bar{C}^{T}(\rho) \bar{C}(\rho)<0
\end{aligned}
$$


Necessity and sufficient conditions for the solutions existence of above inequalities are a quadratic stability of plant $G(\rho)$ [9]. Assume that there exist nonsingular matrices $T_{1}$ and $T_{2}$ such that,

$$
T_{1} U_{1} T_{1}^{T}=T_{1}^{-T} V_{1} T_{1}^{-1}=\Sigma_{g}=\operatorname{diag}\left(\xi_{1}, \xi_{2}, \cdots, \xi_{n}\right), \quad \xi_{1} \geq \xi_{2} \geq \cdots \geq \xi_{n}
$$

and

$$
T_{2} U_{2} T_{2}^{T}=T_{2}^{-T} V_{2} T_{2}^{-1}=\Sigma_{k}=\operatorname{diag}\left(\Sigma_{k 1}, \Sigma_{k 2}\right)
$$

with $\Sigma_{k 1}=\operatorname{diag}\left(\sigma_{1}, \ldots, \sigma_{r}\right), \Sigma_{k 2}=\operatorname{diag}\left(\sigma_{r+1}, \ldots, \sigma_{m}\right), \sigma_{r}>\sigma_{r+1}$ and $\sigma_{j} \geq \sigma_{j+1}$, $j=1,2, \ldots, m$ are $\mathcal{Q}$-singular values of $K(\rho)$. The balanced realization $K(\rho)$ can be written as

$$
\begin{aligned}
K(\rho) & =\left[\begin{array}{l|l}
T_{2} A_{K}(\rho) T_{2}^{-1} & T_{2} B_{K}(\rho) \\
\hline C_{K}(\rho) T_{2}^{-1} & D_{K}(\rho)
\end{array}\right], \\
& =\left[\begin{array}{l|l}
A_{k}(\rho) & B_{k}(\rho) \\
\hline C_{k}(\rho) & D_{k}(\rho)
\end{array}\right], \quad D_{K}(\rho)=D_{k}(\rho), \quad \forall \rho \in \mathcal{F}(\rho) .
\end{aligned}
$$

Further, $K(\rho)$ is partitioned conformably with $\Sigma_{k}=\operatorname{diag}\left(\Sigma_{k 1}, \ldots, \Sigma_{k 2}\right)$ as

$$
K(\rho)=\left[\begin{array}{ll|l}
A_{k 11}(\rho) & A_{k 12}(\rho) & B_{k 1}(\rho) \\
A_{k 21}(\rho) & A_{k 22}(\rho) & B_{k 2}(\rho) \\
\hline C_{k 1}(\rho) & C_{k 2}(\rho) & D_{k}(\rho)
\end{array}\right] .
$$

The truncated realization of $K(\rho)$, denoted by $\hat{K}(\rho)$, has $r$ th-order as follows.

$$
\hat{K}(\rho)=\left[\begin{array}{c|c}
A_{k 11}(\rho) & B_{k 1}(\rho) \\
\hline C_{k 1}(\rho) & D_{k}(\rho)
\end{array}\right], \forall \rho \in \mathcal{F}(\rho) .
$$

Moreover, the reduced-order parameter dependent controller is applied to the plant. We find the reduced parameter dependent closed-loop system

$$
\hat{T}_{\mathrm{wz}}(\rho)=\left[\begin{array}{l|l}
\hat{A}(\rho) & \hat{B}(\rho) \\
\hline \hat{C}(\rho) & \hat{D}(\rho)
\end{array}\right], \forall \rho \in \mathcal{F}(\rho),
$$

where

$$
\begin{aligned}
& \hat{A}=\left[\begin{array}{cc}
A(\rho)+B_{2} D_{k}(\rho) C_{2} & B_{2} C_{k 1}(\rho) \\
B_{k 1}(\rho) C_{2} & A_{k 1}(\rho)
\end{array}\right], \hat{B}=\left[\begin{array}{c}
B_{1}(\rho)+B_{2} D_{k}(\rho) D_{21} \\
B_{k 1}(\rho) D_{21}
\end{array}\right], \\
& \hat{C}=\left[\begin{array}{ll}
C_{1}(\rho)+D_{12} D_{k}(\rho) C_{2} & \left.D_{12} C_{k 1}(\rho)\right], \hat{D}=D_{11}(\rho)+D_{12} D_{k}(\rho) D_{21} .
\end{array}\right.
\end{aligned}
$$




\section{Parameter Dependent Closed-Loop Performance}

In this section, a measure of the degradation of the closed-loop performance caused by applying the reduced-order controller to the high-order parameter dependent plant is derived. We generalize the degradation of the resulted closed-loop system from BT method of LTI systems to parameter dependent system with unbounded-rate parameter. For LTI system the corresponding results can be seen in Zhou, et.al [13]. The next lemma is required to derive an upper bounds for the parameter dependent closed-loop performance degradation.

Lemma 4.1 [12] Assume $G(\rho)$ is an nth-order, quadratically stable, balanced parameter dependent system partitioned conformably with $\Sigma=\operatorname{diag}\left(\Sigma_{1}, \cdots, \Sigma_{2}\right) \geq 0$ as follows.

$$
G(\rho)=\left[\begin{array}{ll|l}
A_{11}(\rho) & A_{12}(\rho) & B_{1}(\rho) \\
A_{21}(\rho) & A_{22}(\rho) & B_{2}(\rho) \\
\hline C_{1}(\rho) & C_{2}(\rho) & D(\rho)
\end{array}\right],
$$

where $\Sigma_{1} \in \mathcal{R}^{r \times r}, \Sigma_{2} \in \mathcal{R}^{n-r \times n-r}$.

Then

$$
\hat{G}(\rho)=\left[\begin{array}{c|c}
A_{11}(\rho) & B_{1}(\rho) \\
\hline C_{1}(\rho) & D(\rho)
\end{array}\right],
$$

is an rth-order, quadratically stable, balanced approximation to $G(\rho)$, and $\|G(\rho)-\hat{G}(\rho)\|_{i, 2} \leq 2 \operatorname{tr}\left(\Sigma_{2}\right)$.

The next theorem is the main result of this section. It is the parameter dependent generalization of the result on time invariant systems.

Theorem 4.1 Suppose $K(\rho)$ is a stabilizing parameter dependent controller such that $T_{z w}(\rho)$ is quadratically stable and there exist Lyapunov inequality solutions $U$ and $V$, such that (7) and (8) are satisfied. Let $\hat{K}(\rho)$ be a reducedorder controller obtained by using BT method. Then the closed-loop system with the reduced-order controller $\hat{K}(\rho)$ is a quadratically stable and $\left\|T_{z w}(\rho)-\hat{T}_{z w}(\rho)\right\|_{i, 2} \leq 2 \sum_{i=r+1}^{m} \sigma_{i}$, where $m$ is the order of the original controller, $r$ is the order of the reduced controller. 


\section{Proof:}

Define $T_{t}=\operatorname{diag}\left(T_{1}, T_{2}\right)$ and $\Sigma_{t}=\operatorname{diag}\left(\Sigma_{t 1}, \Sigma_{t 2}\right)$ with $\Sigma_{t 1}=\operatorname{diag}\left(\xi_{1}, \xi_{2}, \cdots, \xi_{n}, \sigma_{1}, \sigma_{2}, \cdots, \sigma_{r}\right)$ and $\Sigma_{t 2}=\Sigma_{k 2}=\operatorname{diag}\left(\sigma_{r+1}, \cdots, \sigma_{m}\right)$. The parameter dependent closed-loop system with the full-order parameter dependent controller

$$
\bar{T}_{z w}(\rho)=\left[\begin{array}{c|c|c}
T_{t} \bar{A}(\rho) T_{t}^{-1} & T_{t} \bar{B}(\rho) \\
\hline \bar{C}(\rho) T_{t}^{-1} & \bar{D}(\rho)
\end{array}\right]=\left[\begin{array}{c|c}
A_{t}(\rho) & B_{t}(\rho) \\
\hline C_{t}(\rho) & D_{t}(\rho)
\end{array}\right]
$$

is balanced, such that $\forall \rho \in \mathcal{F}_{\rho}$,

$$
\begin{aligned}
& A_{t}(\rho) \Sigma_{t}+\Sigma_{t} A_{t}^{T}(\rho)+B_{t}(\rho) B_{t}^{T}(\rho)<0, \\
& A_{t}^{T}(\rho) \Sigma_{t}+\Sigma_{t} A_{t}(\rho)+C_{t}^{T}(\rho) C_{t}(\rho)<0 .
\end{aligned}
$$

The parameter dependent closed-loop system, $T_{z w}(\rho)$, is partitioned conformably with $\operatorname{diag}\left(\Sigma_{t 1}, \Sigma_{t 2}\right)$ as

$$
\begin{aligned}
& A_{t}(\rho)=\left[\begin{array}{ll}
A_{t 1}(\rho) & A_{t 2}(\rho) \\
A_{t 3}(\rho) & A_{t 4}(\rho)
\end{array}\right] \\
& =\left[\begin{array}{ccc}
T_{1}\left(A(\rho)+B_{2} D_{K}(\rho) C_{2}\right) T_{1}^{-1} & T_{1} B_{2} C_{k 1}(\rho) & T_{1} B_{2} C_{k 2}(\rho) \\
B_{k 1}(\rho) C_{2} T_{1}^{-1} & A_{k 11}(\rho) & A_{k 12}(\rho) \\
B_{k 2} C_{2} T_{1}^{-1} & A_{k 21}(\rho) & A_{k 22}(\rho)
\end{array}\right], \\
& B_{t}=\left[\begin{array}{c}
B_{t 1}(\rho) \\
B_{t 2}(\rho)
\end{array}\right]=\left[\begin{array}{c}
T_{1}\left(B_{1}(\rho)+B_{2} D_{K}(\rho) D_{21}\right) \\
B_{k 1}(\rho) D_{21} \\
B_{k 2}(\rho) D_{21}
\end{array}\right] \\
& C_{t}=\left[\begin{array}{ll}
C_{t 1}(\rho) & C_{t 2}(\rho)
\end{array}\right] \\
& =\left[\left(C_{1}(\rho)+D_{12} D_{K}(\rho) C_{2}\right) T^{-1} \quad D_{12} C_{k 1}(\rho) \quad D_{12} C_{k 2}\right] \\
& D_{t}=D_{11}(\rho)+D_{12} D_{K}(\rho) D_{21} \text {. }
\end{aligned}
$$

Then the realization $\left(A_{t}(\rho), B_{t}(\rho), C_{t}(\rho), D_{t}(\rho)\right)$ can be truncated as 


$$
\hat{T}_{z w}=\left[\begin{array}{cc|c}
T_{1}\left(A(\rho)+B_{2}(\rho) D_{K}(\rho) C_{2}(\rho)\right) T_{1}^{-1} & T_{1} B_{2}(\rho) C_{k 1}(\rho) & T_{1}\left(B_{1}(\rho)+B_{2} D_{K}(\rho) D_{21}\right) \\
B_{k 1}(\rho) C_{2} T_{1}^{-1} & A_{k 11}(\rho) & B_{k 1}(\rho) D_{21} \\
\hline\left(C_{1}(\rho)+D_{12} D_{K}(\rho) C_{2}\right) T_{1}^{-1} & D_{12} C_{k 1}(\rho) & D_{11}(\rho)+D_{12} D_{K}(\rho) D_{21}
\end{array}\right]
$$

and $\hat{T}_{z w}(\rho)$ is a balanced truncation of $T_{z w}(\rho)$ where truncated states correspond to the $\Sigma_{k 2}-\mathcal{Q}$-singular values. Based on the Lemma (4.1), $\hat{T}_{z w}(\rho)$ is a quadratically stable over $\mathcal{P}$ and $\left\|T_{z w}(\rho)-\hat{T}_{z w}(\rho)\right\|_{i, 2} \leq 2 \operatorname{tr} \Sigma_{2}=2 \Sigma_{i=r+1}^{m} \sigma_{i}$.

Solutions $U$ and $V$ are only required to satisfy inequalities (7) and (8), and are known to be not unique. Non-uniqueness of $U, V$ can be exploited to produce more desirable reduced-order controllers. Considering the degradation of the closed-loop performance $\left\|T_{z w}(\rho)-\hat{T}_{z w}(\rho)\right\|_{i, 2} \leq 2 \Sigma_{i=r+1}^{m} \sigma_{i}$ given in Theorem 4.1, it is reasonable to choose $U$ and $V$ such that $\sum_{i=r+1}^{m} \sigma_{i}=\sum_{i=r+1}^{m} \lambda_{i}^{1 / 2}\left(U_{2} V_{2}\right)$ is minimized. However, such solutions are hard to compute since the corresponding optimization is not convex. In this paper we use suboptimal solutions (convex feasibility problems).

Note from (5)-(7) that $A(\rho)+B_{2} D_{K}(\rho) C_{2}$ and $A_{K}(\rho)$ are quadratically stable over $\mathcal{P}$. In particular, when $D_{K}(\rho)=0$ these indicate the parameter dependent plant and controller are quadratically stable. Hence the previous results are not applicable to unstable parameter dependent plant and/or unstable parameter dependent controller. If $G(\rho)$ and/or $K(\rho)$ are not quadratically stable then we use coprime factorization of the parameter dependent controller which is constructed by using feasible solutions of linear matrix inequality to reduce $K(\rho)$ with a procedure as follows.

Define right coprime factorization (RCF) of the $K(\rho)=\mathcal{U}(\rho) \mathcal{V}(\rho)^{-1}$,

$$
\left[\begin{array}{c}
\mathcal{U}(\rho) \\
\mathcal{V}(\rho)
\end{array}\right]=\left[\begin{array}{c|c}
A_{K}(\rho)+B_{K}(\rho) F_{K}(\rho) & B_{K}(\rho) \\
\hline C_{K}(\rho)+D_{K}(\rho) F_{K}(\rho) & D_{K}(\rho) \\
F_{K}(\rho) & I
\end{array}\right]
$$

where $\mathcal{U}(\rho)$ and $\mathcal{V}(\rho)$ are quadratically stable, $F_{K}(\rho)=-B_{K}(\rho)^{T} X$, $X=X^{T}>0$ is a feasible solution of the following inequality: 
$X A_{K}(\rho)+A_{K}(\rho)^{T} X-X B_{K}(\rho) B_{K}(\rho)^{T} X<0, \quad \forall \rho \in \mathcal{F}_{\rho}$. Let $\mathrm{Q}$ and $\mathrm{P}$ are observability and controllability Gramians of RCF, where $P=P^{T}>0$ and $Q=Q^{T}>0$ solve Lyapunov inequalities:

$$
\begin{aligned}
& \left(A_{K}(\rho)+B_{K}(\rho) F_{K}(\rho)\right) P+P\left(A_{K}(\rho)+B_{K}(\rho) F_{K}(\rho)\right)^{T}+B_{K}(\rho) B_{K}(\rho)^{T}<0, \quad \forall \rho \in \mathcal{F}_{\rho} \\
& \left(A_{K}(\rho)+B_{K}(\rho) F_{K}(\rho)\right)^{T} Q+Q\left(A_{K}(\rho)+B_{K}(\rho) F_{K}(\rho)\right)+\left(C_{K}(\rho)+D_{K}(\rho) F_{K}(\rho)\right)^{T} \\
& \left(C_{K}(\rho)+D_{K}(\rho) F_{K}(\rho)\right)+F_{K}(\rho)^{T} F_{K}(\rho)<0, \quad \forall \rho \in \mathcal{F}_{\rho} \text { and }\left[\begin{array}{cc}
P & -\lambda I \\
-\lambda I & Q
\end{array}\right]>0, \lambda>0 .
\end{aligned}
$$

Furthermore, construct balanced $K(\rho)$ by using a state transformation matrix such that the transformed Gramians $P=Q=\Sigma=\operatorname{diag}\left(\sigma_{1}, \cdots, \sigma_{m}\right)$ and then apply balanced truncation method to obtain $\hat{K}(\rho)_{r}=\mathcal{U}(\rho)_{r} \mathcal{V}(\rho)_{r}^{-1}$ which has $r$ th-order, $r<m$.

\section{$5 \quad$ Simulation Results}

In this section we examine the reduced-order controller by applying it to control vertical acceleration of a missile [3]. The missile dynamics under consideration vary greatly as a function of speed $(v)$, angle of attack $(\alpha)$, and altitude $(H)$. The parameter dependent system $G_{\rho}$ is described as follows.

$$
\begin{aligned}
& {\left[\begin{array}{c}
\dot{\alpha} \\
\dot{q}
\end{array}\right]=\left[\begin{array}{cc}
-Z_{\alpha} & 1 \\
-M_{\alpha} & 0
\end{array}\right]\left[\begin{array}{l}
\alpha \\
q
\end{array}\right]+\left[\begin{array}{l}
0 \\
1
\end{array}\right] \delta_{m},} \\
& {\left[\begin{array}{c}
a_{z v} \\
q
\end{array}\right]=\left[\begin{array}{cc}
-1 & 0 \\
0 & 1
\end{array}\right]\left[\begin{array}{l}
\alpha \\
q
\end{array}\right],}
\end{aligned}
$$

where $Z_{\alpha}$ and $M_{\alpha}$ are parameter depending on $v, \alpha$, and $H$. We assume that $Z_{\alpha}, M_{\alpha}$ are measured in real time. The states are angle of attack $(\alpha)$ and pitch rate $(q)$. The input to the model is fin deflection $\left(\delta_{m}\right)$. The output are normalized vertical acceleration $\left(a_{z v}\right)$ and pitch rate $(q)$. Let $v, \alpha$, and $H$ vary in $0.5 \leq v \leq 4$ (mach), $0 \leq \alpha \leq 40$ (degrees), $0 \leq H \leq 18(\mathrm{~km})$ during operation, the coefficients $Z_{\alpha}$ and $M_{\alpha}$ range in $Z_{\alpha} \in[0.5,4]$ and $M_{\alpha} \in[0,106]$. Note that $Z_{\alpha}$ and $M_{\alpha}$ enter the states space matrices in an affine way and it can be considered as representing a convex polytope with four vertices. The aim of the controller design is to achieve the step response of the vertical acceleration $\left(a_{z v}\right)$ having settling time $<0.5$ seconds for all variations of $Z_{\alpha}$ and $M_{\alpha}$ and 
overshoot limitation is $15 \%$. The selection of weights is based on a frozen-time analysis of the parameter dependent systems, and follows the same lines as $\mathcal{H}_{\infty}$. The weights must enforce adequate settling time 0.5 seconds and highfrequency gain attenuation $[3,4]$. We choose the sensitivity weight $W_{1}$ and the robustness weight $W_{2}$. The performance and robustness requirements correspond to controller design which satisfy

$$
\left\|\begin{array}{c}
W_{1}\left(I+G_{\rho} K_{\rho}\right)^{-1} \\
W_{2} K_{\rho}\left(I+G_{\rho} K_{\rho}\right)^{-1}
\end{array}\right\|_{\infty}<1,
$$

where

$$
\begin{aligned}
& W_{1}=\frac{2.01}{s+0.201}, \\
& W_{2}=\frac{96.78 s^{3}+0.29 s^{2}+0.0003 s-0.0039}{s^{3}+1.12 \times 10^{5} s^{2}+1.05 \times 10^{8} s+1.07 \times 10^{11}} .
\end{aligned}
$$

The high-order plant having 6th-order consists of the missile dynamic and all weighting functions. The full-order parameter dependent controller is designed by using procedure developed by Apkarian[2]. The resulting parameter dependent controller has the same order as that of the high-order plant. The feasibility problems (7) and (8) are convex problems. Using LMI Control toolbox for MATLAB[8] in pentium(R) 4, $2400 \mathrm{MHz}, 18 \mathrm{x}, 512 \mathrm{MB}$ of RAM we obtain feasible solutions $U$ and $V$ after 78 iterations (corresponds to CPU time of 2.157 seconds). Furthermore, the order of the balanced parameter dependent controller is reduced by using the BT method suggested in the preceding section. The upper bound of the parameter dependent closed-loop performance for all variations of parameters is 0.2669. At 18 frozen parameter

\begin{tabular}{|c|c|c|c|c|c|c|c|c|c|c|c|c|c|}
\hline & \multicolumn{3}{|c|}{ 6th-order } & \multicolumn{3}{|c|}{ 5th-order } & \multicolumn{3}{|c|}{ 4th-order } & \multicolumn{3}{|c|}{ 3rd-order } \\
\hline & & \multicolumn{3}{|c|}{$Z_{\alpha}$} & \multicolumn{3}{|c|}{$Z_{\alpha}$} & \multicolumn{3}{|c|}{$Z_{\alpha}$} & \multicolumn{3}{|c|}{$Z_{\alpha}$} \\
\hline & & 0.5 & 2 & 3.5 & 0.5 & 2 & 3.5 & 0.5 & 2 & 3.5 & 0.5 & 2 & 3.5 \\
\hline \multirow{6}{*}{$M_{\alpha}$} & 0 & 0.119 & 0.117 & 0.116 & 0.119 & 0.117 & 0.181 & 0.229 & 0.521 & 0.732 & 0.299 & 1.152 & 1.932 \\
\hline & 20 & 0.118 & 0.116 & 0.116 & 0.118 & 0.116 & 0.117 & 0.443 & 0.640 & 0.827 & 1.284 & 2.022 & 2.700 \\
\hline & 40 & 0.117 & 0.116 & 0.116 & 0.117 & 0.116 & 0.117 & 0.354 & 0.718 & 0.899 & 2.105 & 2.756 & 3.347 \\
\hline & 60 & 0.116 & 0.116 & 0.117 & 0.116 & 0.116 & 0.117 & 0.599 & 0.782 & 0.962 & 2.793 & 3.369 & 3.898 \\
\hline & 80 & 0.116 & 0.116 & 0.117 & 0.116 & 0.116 & 0.118 & 0.659 & 0.842 & 1.018 & 3.395 & 3.912 & 4.378 \\
\hline & 100 & 0.115 & 0.116 & 0.118 & 0.116 & 0.116 & 0.118 & 0.716 & 0.896 & 1.074 & 3.910 & 4.368 & 4.797 \\
\hline
\end{tabular}
values, the $\mathcal{H}_{\infty}$-norm of the parameter dependent closed loop systems with some reduced-order controllers are shown in Table 1.

Table 1 The frozen parameter dependent closed loop performance. 
When the full-order controller is reduced to 3rd order, $\mathcal{H}_{\infty}$-norm of the closed loop systems turns out be much greater than $\mathcal{H}_{\infty}$-norm of the closed loop with full-order controller.

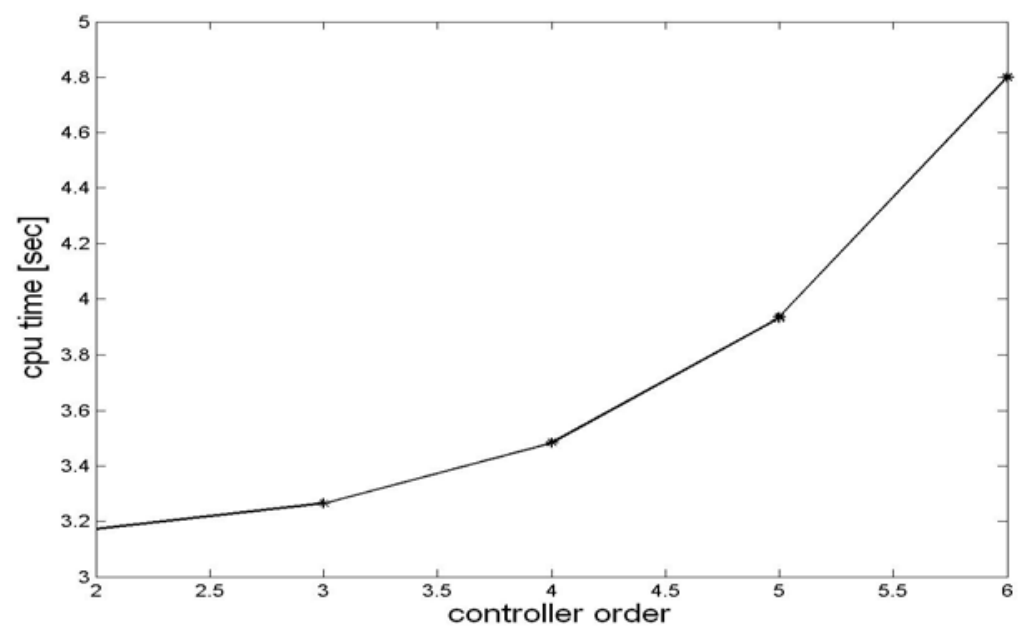

Figure 1 CPU time versus controller order.

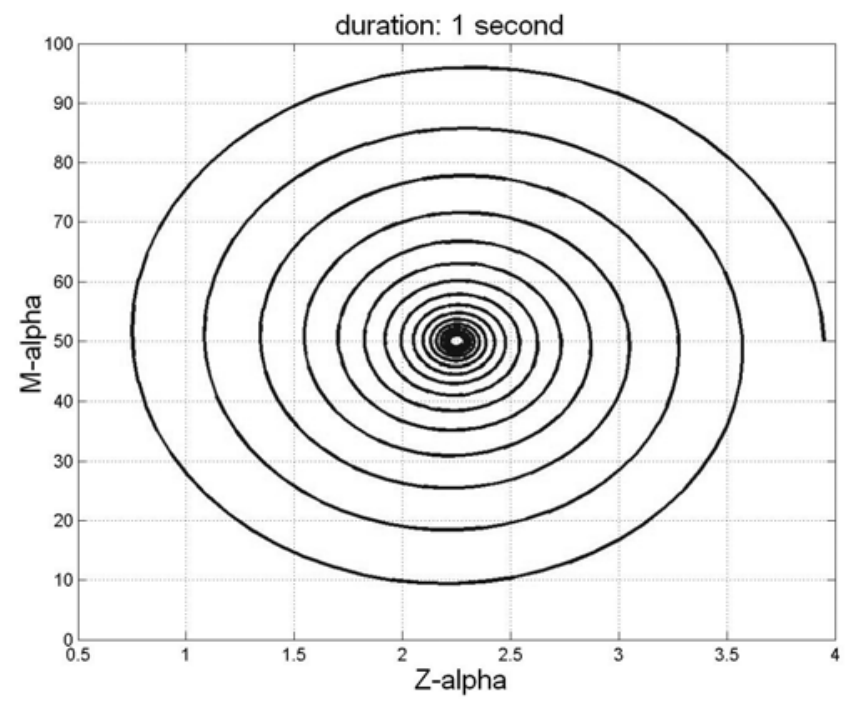

Figure 2 Parameter trajectory. 

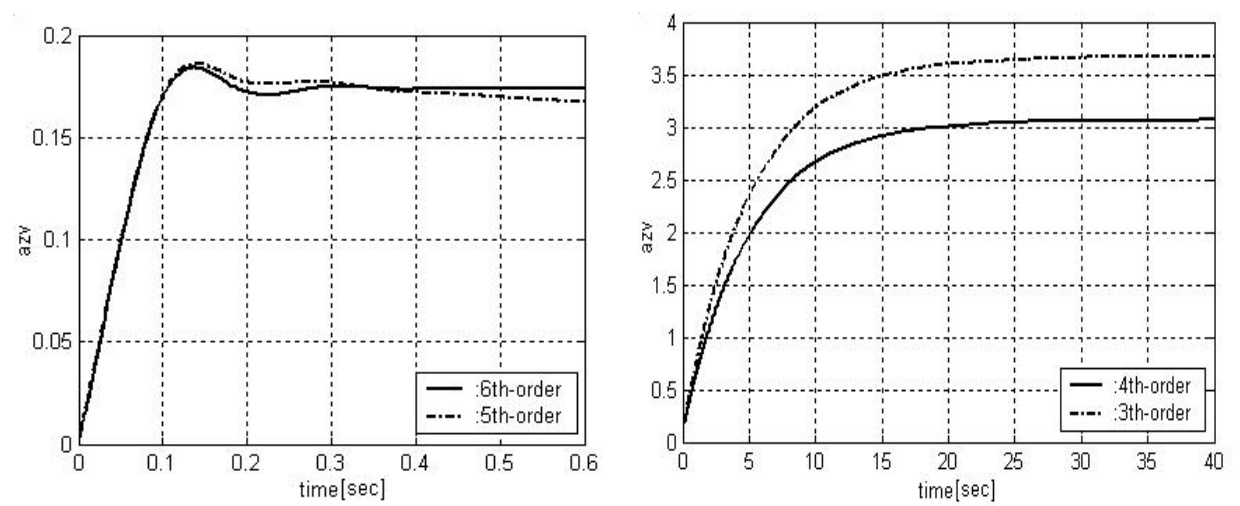

Figure 3 Time responses of the parameter dependent closed-loop systems.

To provide an indication of the average time required for the proposed controller reduction method and the increase in complexity with the controller order, the CPU time required for finding reduced-order controllers is shown in Figure 1. To analyze the stability and performance robustness of the closed-loop systems, we use the particular spiral parameter trajectory [12] as follows.

$$
\begin{aligned}
& Z_{\alpha}(t)=2.25+1.70 e^{-4 t} \cos (100 t), \\
& M_{\alpha}(t)=50+49 e^{-4 t} \sin (100 t) .
\end{aligned}
$$

This trajectory is given in Figure 2. To conform the quadratic stability of the full-and reduced-closed loop system, the solution $P$ satisfying inequality (2) is found after 47, 37, 30, 30 iterations for 6th-, 5th-, 4th-, 3rd-order controllers respectively. The step responses of the closed-loop system with the 6th-, 5th-, 4th-, and 3rd-order parameter dependent controllers for all parameter trajectories are described in Figure 3.

From these figures, it can be seen that the settling time of the 6th- and 5th-order controller are lower than 0.5 seconds and the closed-loop step responses achieve stability within 0.4 seconds for all parameter trajectories. When the parameter dependent controller is reduced to as low as 4th- and 3rd-order, the parameter dependent closed-loop systems are quadratically stable, although the settling time of its step responses are much greater than 0.5 seconds.

\section{Conclusion}

In this paper, the extension of the balanced truncation to reduce the order of the parameter dependent controller is proposed. When the parameter dependent plant and controller are quadratically stable, the degradation of the closed-loop performance caused by applying the reduced-order controller is bounded by twice the sum of the truncated $\mathcal{Q}$-singular values. The proposed method has 
been applied to a missile model. From the simulation results, the step response of the parameter dependent closed-loop performance with 5th-order controller maintains the stability and performance of the full-order controller for all parameter trajectories.

\section{Acknowledgement}

We would like to thank the reviewers for their detailed comments and suggestions.

\section{References}

1. Anderson, B. D. O., Controller Design: Moving from Theory to Practice, IEEE Control System, 16-25 (1993).

2. Apkarian, P. \& Adam, R. J., Advanced Gain Scheduling Techniques for Uncertain System, Proceeding American Control Conference (1997).

3. Apkarian, P., Gahinet, P. \& Becker, G., Self-Scheduled $\mathcal{H}_{\infty}$ Control of Linear Parameter Varying Systems: a Design Example\}, Automatica 31, 9, 1251-1261 (1995).

4. Riyanto, B., Self-Scheduled $\mathcal{H}_{\infty}$ Control Design for N250 Aircraft Dynamics Based on Polytopic LPV Model, Proc. of the IASTED International Conference Control and Applications, Banff, Canada, pp. 52-57 (1999).

5. $\quad$ Becker, G. S., Quadratic Stability and Performance of Linear Parameter Varying Dependent Systems, Ph.D Dissertation, University of California at Berkeley (1993).

6. El-Zobaidi, H. M. H. \& Jaimoukha, I., Robust Control and Model and Controller Reduction of Linear Parameter Varying Systems, Proc. of the 37th IEEE Conference on Decision and Control, Tampa, Florida USA 3, 3015-3020 (1998).

7. Enns, D. F., Model reduction with balanced realizations: an error bound and a frequency weighted generalization\}, Proceedings of 23rd Conference on Decision and Control Las Vegas, NV, 127-132 (1984).

8. Gahinet, P., Nemirovski, A., Laub, A. \& Chilali, M., The LMI Control Toolbox, The MathWorks, Inc.: Natick, MA (1995).

9. Goddard, P. J., Performance-Perserving Controller Approximation, PhD Dissertation, Trinity College Cambridge (1995).

10. Lall, S. and Beck, C., Error-Bounds for Balanced Model-Reduction of Linear Time-Varying Systems, IEEE Transactions on Automatic Control \{lbf 48\}, 6, 946-956 (2003).

11. Sandberg, H. \& Rantzer, A., Balanced Model Reduction of Linear TimeVarying Systems, IFAC World Conggress (2002). 
12. Wood, G. D., Goddard, P. J., \& Glover, K., Approximation of Linear Parameter-Varying Systems, Proceedings of 35th Conference on Decision and Control (1996).

13. Zhou, K., D'Souza, C. \& Cloutier, J. R., Structurally Balanced Controller Order Reduction with Guaranteed Closed Loop Performance, System and Control letters 24, 235-242 (1995). 